\title{
On the spectral functions of scalar mesons
}

\author{
Francesco Giacosa and Giuseppe Pagliara \\ Institut für Theoretische Physik, Universität Frankfurt, \\ Johann Wolfgang Goethe - Universität, Max von Laue-Str. 160438 Frankfurt, Germany
}

\begin{abstract}
In this work we study the spectral functions of scalar mesons in one- and two-channel cases. When the propagators satisfy the Källen-Lehman representation a normalized spectral function is obtained, allowing to take into account finite-width effects in the evaluation of decay rates. In the one-channel case, suitable to the light sigma and $\mathrm{k}$ mesons, the spectral function can deviate consistently from a Breit-Wigner shape. In the two-channel case with one subthreshold channel the evaluated spectral function is well approximated by a Flatte' distribution; when applying the study to the $a_{0}(980)$ and $f_{0}(980)$ mesons the tree-level forbidden KK decay is analysed.
\end{abstract}

\section{INTRODUCTION}

The scalar mesons below $2 \mathrm{GeV}$ are in center of debate since many years [1, 2, 3, 4]. More states than expected from the quark-antiquark assignment are reported in Particle Data Group (PDG) [5], leading to the introduction of a scalar glueball [6], tetraquark states [7] and mesonic molecules [8]. In particular, the scalar resonances below 1 $\mathrm{GeV}$ have appealing characteristics, such as the reversed level ordering of masses, expected from tetraquark states $[3,7,9,10,11]$. In turn, this scenario implies that quarkonia lie between 1 and $2 \mathrm{GeV}$. A complication in the analysis of scalar states is mixing: between 1-2 GeV a quarkonia-glueball mixing in the isoscalar sector is considered, for instance, in Refs. 12]. Mixing among tetraquark states below $1 \mathrm{GeV}$ and quarkonia above $2 \mathrm{GeV}$ is studied in Refs. [13, 14, 15], where, however, the results do not coincide: while a large mixing is found in Ref. [14], a negligible mixing is the outcome of [15]. It should be stressed that different interpretations of scalar state are possible: a nonet of scalar quarkonia is settled below $1 \mathrm{GeV}$ in Refs. [16] in agreement with the linear sigma model and the Nambu Jona-Lasinio (NJL) model, while in Ref. [17] a broad glueball, to be identified with $f_{0}(600)$, is proposed. We refer to Refs. [1, 2, 3, 10] for a discussion of arguments in favour and/or against the outlined assignments.

Studies on scalar mesons have been extensively performed by using chiral perturbation theory [18], where a scalar resonance at about $440 \mathrm{MeV}$ is inferred out of pion-pion scattering. A full nonet of molecular-like scalar states is generated in the unitarized chiral perturbation theory of Ref. [19]. In particular, Pelaez [20] studied the large- $N_{c}$ dependence of the light scalar resonances finding that they do not scale as quarkonia but in agreement with a molecular or tetraquark composition (see, however, also the discussions in Refs. 21]).

In the present paper we concentrate on an important aspect of light scalar resonances, namely the form of their spectral functions, in a simple theoretical context. In this study, relevant for both quarkonium or tetraquark assignment of light scalars 22], effects arising from loops of pseudoscalar mesons are considered: this leads to parametrizations of spectral functions beyond the (usually employed) Breit-Wigner and Flattè distributions and allow to include finitewidth effects in the evaluation of decay rates. In particular, we consider the following physical scenarios: (i) the case of a broad scalar resonance, strongly coupled to one decay channel, such as the $\sigma \equiv f_{0}(600)$ in the pion-pion decay mode, for which the spectral function can deviate substantially form the Breit-Wigner form; (ii) the case of two channels, one of which is sub-threshold for the mass and thus forbidden at tree-level, as the $\bar{K} K$ decay mode for the resonances $f_{0}(980)$ and $a_{0}(980)$. In the latter case a comparison with the usually employed Flattè distribution is performed.

The key-quantity of the discussion is the propagator of scalar resonances dressed by mesonic loops in one or more channels. When the Källen-Lehmann representation is satisfied, as verified at one-loop level in the case of light scalar mesons for large ranges of parameters [22], the spectral function (proportional to the imaginary part of the propagator) is correctly normalized and is interpreted as a 'mass distribution' for the scalar state. A general definition of the decay of a scalar state, involving the obtained mass distribution, is then possible. In this way one takes into account in a consistent fashion finite-width effects for the decay, hence allowing to study deviations form the usually employed tree-level formula for decay rates. Furthermore, the fulfillment of the Källen-Lehmann representation offers a criterion to delimit the validity of our one-loop study: as soon as violations appear (generally for large coupling constants) the obtained spectral functions are no longer usable.

We regularize the mesonic loops by means of a cutoff function which in turn is equivalent to a nonlocal interaction Lagrangian. In a phenomenological perspective it is reasonable that the mesonic states in the loop cannot have indefinitely high virtual momenta which are naturally limited due to the finite range of the meson-meson interaction. We also show that the dependence on the chosen cutoff function and on the specific value of the cutoff is mild.

In order to render the paper easily understandable and self-contained we start in Section II with one-channel case by recalling the basic definitions and properties, then we apply the study to the scalar sigma $\sigma$ and kaon $k$ resonances: 
the corresponding spectral function shows consistent deviations from the usual Breit-Wigner one. In Section III we turn to the two-channel case, with particular attention to the resonances $a_{0}(980)$ and $f_{0}(980)$, their decay rates and spectral functions in comparison with the Flattè distribution 23, 24]. Implications of the results in view of a nonet of tetraquark states below $1 \mathrm{GeV}$ is discussed. In section IV we drive our conclusions, emphasizing as in Ref. 22. that the use of propagators fulfilling the Källen-Lehmann representation, which implies normalized distributions and a correct definition of decay rates, should be preferable both in theoretical and experimental works.

\section{SCALAR SPECTRAL FUNCTION: ONE-CHANNEL CASE}

\section{A. Definitions and properties}

We consider the scalar fields $S$ and $\varphi$ described by the Lagrangian

$$
\mathcal{L}_{S}^{1}=\frac{1}{2}\left(\partial_{\mu} S\right)^{2}-\frac{1}{2} M_{0}^{2} S^{2}+\frac{1}{2}\left(\partial_{\mu} \varphi\right)^{2}-\frac{1}{2} m^{2} \varphi^{2}+g S \varphi^{2} .
$$

In the limit $g=0$ the propagator of the field $S$ reads

$$
\Delta_{S}(p)=\frac{1}{p^{2}-M_{0}^{2}+i \varepsilon}, g=0 .
$$

We intend to study the modification to $\Delta_{S}(p)$ when $g \neq 0$, which arises by considering the loop-diagram of Fig. 1 and how this contribution affects the decay mechanism $S \rightarrow \varphi \varphi$. We recall that at tree-level the decay width reads

$$
\Gamma_{S \varphi \varphi}^{\mathrm{t}-1}\left(M_{0}\right)=\frac{p_{S \varphi \varphi}}{8 \pi M_{0}^{2}}\left[g_{S \varphi \varphi}\right]^{2} \theta\left(M_{0}-2 m\right), p_{S \varphi \varphi}=\sqrt{\frac{M_{0}^{2}}{4}-m^{2}}, g_{S \varphi \varphi}=\sqrt{2} g .
$$

where $\theta(x)$ is the step function and

(The factor $\sqrt{2}$ in the amplitude $g_{S \rightarrow \varphi \varphi}$ takes into account that the final state consists of two identical particles.) In general, under the symbol $p_{S A B}$ the expression

$$
p_{S A B}=\frac{1}{2 M_{S}} \sqrt{M_{S}^{4}+\left(M_{A}^{2}-M_{B}^{2}\right)^{2}-2\left(M_{A}^{2}+M_{B}^{2}\right) M_{S}^{2}}
$$

i.e. the momentum of the outgoing particle(s), is understood.

At tree-level the particle $S$ is treated as stable. However, the very fact that the decay $\Gamma_{S \varphi \varphi}^{\mathrm{t}-\mathrm{l}} \neq 0$ for $M_{0}>2 \mathrm{~m}$ means that $S$ is not stable and cannot be considered as an asymptotic state of the Lagrangian $\mathcal{L}_{S}^{1}$. The tree-level expression $\Gamma_{S \varphi \varphi}^{\mathrm{t}-\mathrm{l}}$ is only valid in the limit $g \rightarrow 0$. The evaluation of the loop of Fig. 1 offers a way to define and interpret the decay $S \rightarrow \varphi \varphi$ as we describe in the following. The modified propagator of $S$ is obtained by (re)summing the loop-diagrams of Fig. 1:

$$
\Delta_{S}\left(p^{2}\right)=\frac{1}{p^{2}-M_{0}^{2}+g_{S \varphi \varphi}^{2} \Sigma\left(p^{2}\right)+i \varepsilon}
$$

where the self-energy $\Sigma\left(p^{2}\right)$ reads:

$$
\Sigma\left(p^{2}\right)=-i \int \frac{d^{4} q}{(2 \pi)^{4}} \frac{1}{\left[(q+p / 2)^{2}-m^{2}+i \varepsilon\right]\left[(q-p / 2)^{2}-m^{2}+i \varepsilon\right]} .
$$

The integral defining $\Sigma\left(p^{2}\right)$ is, as known, logarithmic divergent. Our intention is to consider the Lagrangian $\mathcal{L}_{S}^{1}$ as an effective low-energy description of the fields $S$ and $\varphi$, and not as a fundamental theory valid up to indefinitely high mass scales. We do not apply the renormalization scheme to $\mathcal{L}_{S}^{1}$ but we introduce a regularization function $f_{\Lambda}(q)$ which depends on a cut-off scale $\Lambda$ for the large momenta. The self-energy $\Sigma\left(p^{2}\right)$ is then modified to:

$$
\Sigma\left(p^{2}\right)=-i \int \frac{d^{4} q}{(2 \pi)^{4}} \frac{f_{\Lambda}^{2}\left(q^{o}, \vec{q}\right)}{\left[(q+p / 2)^{2}-m^{2}+i \varepsilon\right]\left[(q-p / 2)^{2}-m^{2}+i \varepsilon\right]}
$$

When choosing $f_{\Lambda}(q)=f_{\Lambda}\left(q^{2}\right)$ the covariance of the theory is preserved, otherwise is lost. Indeed, in many calculations related to mesonic loops a regularization of the kind $f_{\Lambda}(q)=f_{\Lambda}\left(\vec{q}^{2}\right)$ is chosen, which leads to simple expressions 


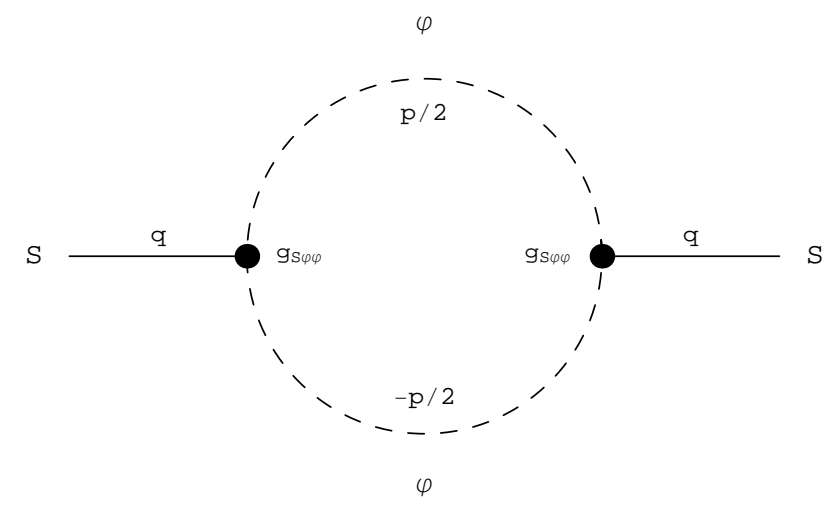

FIG. 1: Mesonic loop

for the self-energy contribution but breaks covariance explicitly, and thus is strictly valid only in the rest frame of the decaying particle. In particular, the 3d-cutoff $f_{\Lambda}\left(\vec{q}^{2}\right)=\theta\left(\Lambda^{2}-\vec{q}^{2}\right)$ is often used. We refer to Appendix A for a closer analysis of the self-energy $\Sigma\left(p^{2}\right)$, where the case of unequal masses circulating in the loop is also presented. The interaction strength among light mesons is suppressed for distances larger than $l \sim 0.5-1 \mathrm{fm}$ : in this particular physical example it is then natural to implement a cutoff $\Lambda \sim 1 / l$, which varies between 1 and $2 \mathrm{GeV}$.

The cutoff-function $f_{\Lambda}(q)$ is not present in the Lagrangian $\mathcal{L}_{S}^{1}$ of Eq. (1). In this sense the Lagrangian is incomplete because it does not specify how to cut the high momenta. One can take into account $f_{\Lambda}(q)$ already at the Lagrangian level by rendering the interaction term nonlocal:

$$
\left(\mathcal{L}_{S}^{1}\right)_{i n t}=g S \varphi^{2} \rightarrow g S \int d^{4} y \varphi(x+y / 2) \varphi(x-y / 2) \Phi(y) .
$$

The Feynman rule for the 3-leg vertex is modified as:

$$
i g \rightarrow i g f_{\Lambda}\left(\frac{q_{1}-q_{2}}{2}\right), f_{\Lambda}(q)=\int d^{4} y \Phi(y) e^{-i y q}
$$

where $q_{1}$ and $q_{2}$ are the momenta of the two particles $\varphi$. The function $f_{\Lambda}(q)$ enters directly into the expression of all amplitudes. In particular, the self-energy contribution of Eq. (7) is now obtained by application of the (modified) Feynman rules to the loop diagram of Fig. 1. Indeed, the delocalization of the interaction term also induces a change of the tree-level result for the decay, which becomes (for the case $f_{\Lambda}(q)=f_{\Lambda}\left(\vec{q}^{2}\right)$ ):

$$
\Gamma_{S \varphi \varphi}^{\mathrm{t}-1}\left(M_{0}\right)=\frac{p_{S \varphi \varphi}}{8 \pi M_{0}^{2}}\left[g_{S \varphi \varphi} f_{\Lambda}\left(\vec{q}^{2}=p_{S \varphi \varphi}^{2}\right)\right]^{2} \theta\left(M_{0}-2 m\right), g_{S \varphi \varphi}=\sqrt{2} g
$$

that is the function $f_{\Lambda}\left(\vec{q}^{2}\right)$ is explicitly present in the tree-level decay expression and can be interpreted as a phenomenological form factor [27]

If a step-function is used the local tree-level expression of Eq. (3) is recovered, provided that the cutoff $\Lambda$ is large enough. In this work we use the following cutoff-function

$$
f_{\Lambda}(q)=f_{\Lambda}\left(\vec{q}^{2}\right)=\left(1+\vec{q}^{2} / \Lambda^{2}\right)^{-1} .
$$

With this choice the Fourier transform $\Phi(y)$, see Eq. (9), takes the form $\delta\left(y^{0}\right) \exp [-|\vec{y}| \Lambda] /|\vec{y}|$, thus decreasing rapidly for increasing distance of the two interacting mesons $\varphi$. The interaction range $l$ is of the order $\Lambda^{-1}$, as discussed above based on general dimensional grounds. At each step of the forthcoming study we employed also different forms of $f_{\Lambda}(q)$, finding that the dependence on the precise form of $f_{\Lambda}(q)$ affects only slightly the results. Notice that in Refs. [25] a similar equation to (10) (where $s=\vec{q}^{2}$ and $f_{\Lambda}=G(s)$ in the notation of [25]) represents the starting point of the analysis. The function $G(s)$ in the above cited works is taken to be a Gaussian, $\Lambda$ is of the order of $1 \mathrm{GeV}$. The present approach shows the link between such form factor $f_{\Lambda}=G(s)$ and a nonlocal Lagrangian. However, we will not concentrate as in [25] on scattering amplitudes but on spectral functions and decay widths. At the same time we do not relate the imaginary and real part of the propagator via Källen-Lehmann dispersion relation, but we evaluate them independently and subsequently we check numerically if it satisfied, see details in the following discussion. 


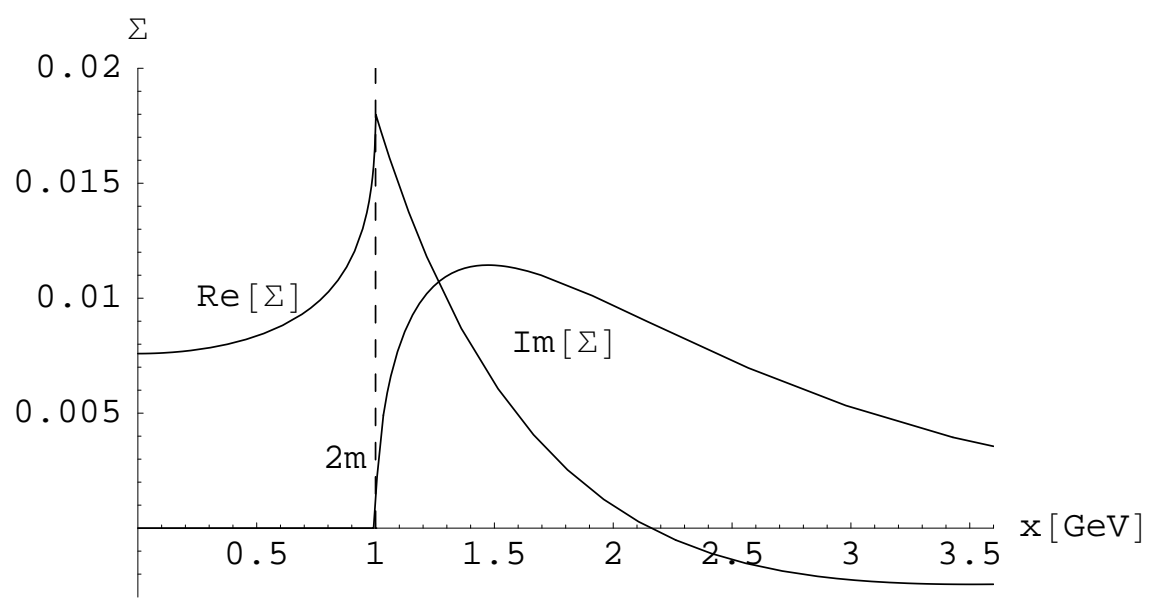

FIG. 2: Real and imaginary part of the mesonic loop for $m=0.5 \mathrm{GeV}$ and $\Lambda=1.5 \mathrm{GeV}$.

Let us now turn to the self-energy $\Sigma\left(p^{2}\right)$. A general property for $\Sigma\left(p^{2}\right)$ follows from the optical theorem:

$$
I(x)=g_{S \phi \phi}^{2} \operatorname{Im}\left[\Sigma\left(x=\sqrt{p^{2}}\right)\right]=x \Gamma_{S \phi \phi}^{\mathrm{t}-1}(x) .
$$

The imaginary part of the self-energy diagram is zero for $0<x<2 m$ and nonzero starting at threshold. The real part

$$
R(x)=g_{S \varphi \varphi}^{2} \operatorname{Re}\left[\Sigma\left(x=\sqrt{p^{2}}\right)\right]
$$

is nonzero below and above threshold. In Fig. 2 the functions $R(x)$ and $I(x)$ are plotted using Eq. (11). A particular choice for the parameters $m=0.5 \mathrm{GeV}$ and $\Lambda=1.5 \mathrm{GeV}$ (of the order of physical cases studied later) is done. Anyway the plotted functions are qualitatively similar for large ranges of parameters. As noticeable, $R(x)$ is continuous but not derivable in $x=2 m$. It has a cusp at $x=2 m$ : the left derivative is $+\infty$, while the right derivative is finite and negative.

In terms of the two functions $R(x)$ and $I(x)$ the propagator of Eq. (2) reads

$$
\Delta_{S}(x)=\frac{1}{x^{2}-M_{0}^{2}+R(x)+i I(x)+i \varepsilon} .
$$

We define the (Breit-Wigner) mass $M$ for the scalar field $S$ as the solution of the equation

$$
M^{2}-M_{0}^{2}+R(M)=0
$$

When the function $R(M)$ is positive, which is usually the physical case (Fig. 2), the dressed mass $M$ is smaller than the bare mass $M_{0}$, showing that the quantum fluctuations tend to lower it.

We now turn to the spectral function $d_{S}(x)$ of the scalar field $S$ related to the imaginary part of the propagator as

$$
d_{S}(x)=\frac{2 x}{\pi}\left|\lim _{\varepsilon \rightarrow 0} \operatorname{Im}\left[\Delta_{S}(x)\right]\right|
$$

In the limit $g \rightarrow 0$ we obtain the desired spectral function $d_{S}(x)=\delta\left(x-M_{0}\right)$. The normalization of $d_{S}(x)$ holds for each $g$

$$
\int_{0}^{\infty} d_{S}(x) d x=1 .
$$

The latter Eq. is a consequence of the Källen-Lehmann representation

$$
\Delta_{S}(x)=\int_{0}^{\infty} d y \frac{2 y}{\pi} \frac{-\operatorname{Im}\left[\Delta_{S}(y)\right]}{y^{2}-x^{2}+i \varepsilon}
$$




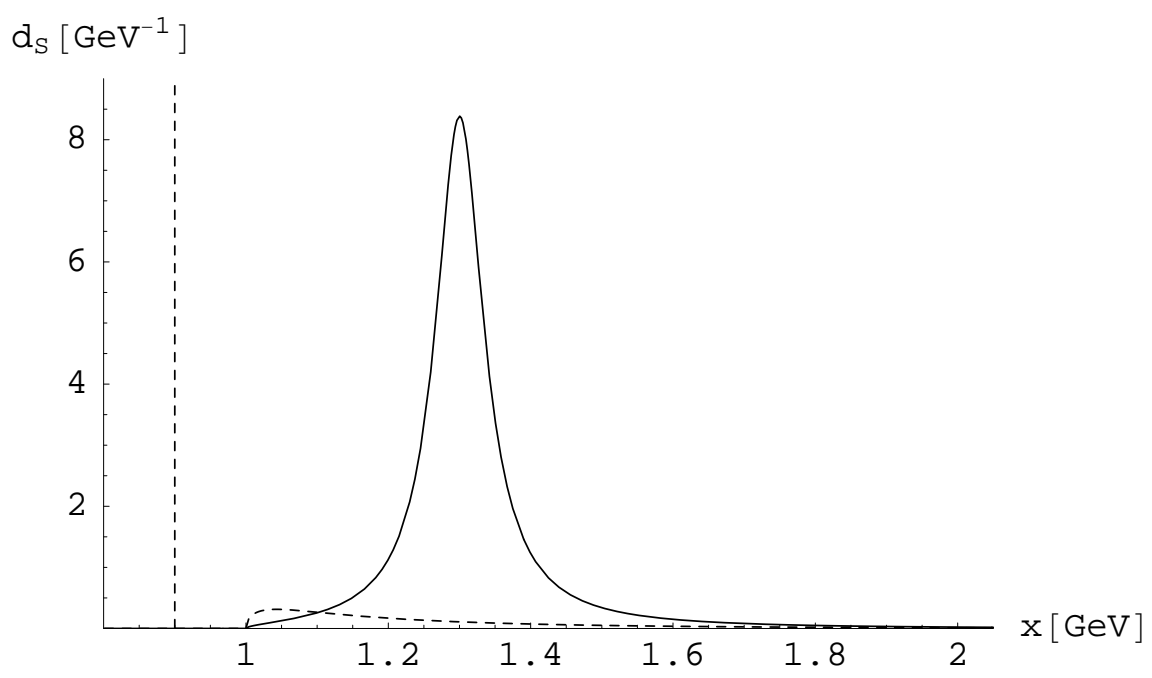

FIG. 3: Spectral functions in the cases $M<2 m$ (dashed line) and $M>2 m$ (continuous line). The coupling constant is $g_{S \phi \phi}=3 \mathrm{GeV}$ and two values of the masses are chosen: $M=0.9 \mathrm{GeV}$ and $M=1.3 \mathrm{GeV}, m=0.5 \mathrm{GeV}$.

when taking the limit $x \rightarrow \infty$. Eqs. (17) and (18) hold in general for the full propagator. In our case we check numerically the validity of the normalization condition (17) at one-loop level of Fig. 1. We find that it is fulfilled to a high level of accuracy for large ranges of parameters, see also the discussion in [22] and in the next subsection.

Let us consider $d_{S}(x)$ in the two interesting cases $M<2 m$ and $M>2 m$. If $M<2 m$ Eq. (16) becomes:

$$
d_{S}(x)=Z \delta(x-M) \theta(2 m-x)+\frac{2 x}{\pi} \frac{I(x)}{\left(x^{2}-M_{0}^{2}+R(x)\right)^{2}+I(x)^{2}}
$$

where

$$
Z=\left(1+\frac{1}{2 M}\left(\frac{d R}{d x}\right)_{x=M}\right)^{-1}
$$

When $M<2 m$ the constant $Z$ is usually reabsorbed into the definition of the wave function renormalization, hence recovering the free propagator properly normalized as $\left(p^{2}-M^{2}+i \varepsilon\right)^{-1}$, corresponding to $d_{S}(x)=\delta(x-M)$ for $x<2 m$ as in the free case $g=0$. Thus, we have still a stable particle with dressed mass $M$ instead of $M_{0}$. Notice that $0<Z<1$ because $R^{\prime}(M)$ is a positive number: the quantity $(1-Z)$ can be interpreted as the amount of virtual clouds of $2 \varphi$ contributing to the wave-function.

If $M>2 m$ the spectral function reads

$$
d_{S}(x)=\frac{2 x}{\pi} \frac{I(x)}{\left(x^{2}-M_{0}^{2}+R(x)\right)^{2}+I(x)^{2}} .
$$

No delta-functions are present but typically a picked distribution $d_{S}(x)$ is obtained, corresponding to a physical resonance. The mass $M$ is not the maximum of the $d_{S}(x)$ although in general very close to it. Consistent deviations can appear when $M$ is close to threshold and for large coupling constant, see next subsection for a more detailed discussion of this point. Notice moreover that $d_{S}(x)$ is zero for $x<2 m$.

We plot the typical behavior of the spectral function in both cases $M<2 m$ and $M>2 m$ in Fig. [3. We used the values $M=0.9 \mathrm{GeV}$ and $M=1.3 \mathrm{GeV}$ corresponding to the the two cases below and above the threshold, $m=0.5$ $\mathrm{GeV}$ as before and $g_{S \phi \phi}=3 \mathrm{GeV}$. The value of $Z$, for the sub-threshold case, is $\sim 0.9$, we have numerically verified that the spectral functions are normalized in both cases.

When $M>2 m$ the function $d_{S}(x)$ can be interpreted as the mass distribution of the resonance, see also Appendix B for an intuitive discussion about this point. We then define the decay rate for the process $S \rightarrow \varphi \varphi$ by implementing the distribution $d_{S}(x)$, and thus including finite width effects, as:

$$
\Gamma_{S \varphi \varphi}=\int_{0}^{\infty} d x d_{S}(x) \Gamma_{S \varphi \varphi}^{\mathrm{t}-1}(x) .
$$


This formula reduces to the tree-level amplitude $\Gamma_{S \varphi \varphi}^{\mathrm{t}-1}\left(M_{0}\right)$ of Eq. (10) in the limit of small $g$ :

$$
\Gamma_{S \varphi \varphi}^{\mathrm{t}-\mathrm{l}}\left(M_{0}\right) \simeq \Gamma_{S \varphi \varphi} \text { for } g \rightarrow 0 .
$$

Notice that in this limit $M \rightarrow M_{0}$. However, even for finite $g$, when $M \neq M_{0}$, the formula $\Gamma_{S \varphi \varphi} \simeq \Gamma_{S \varphi \varphi}^{\mathrm{t}-\mathrm{l}}(M)$ offers a first approximation to the decay width of the state as long as the distribution is picked, i.e. the scalar state $S$ is not too broad.

The definition Eq. (22) for the decay $S \rightarrow \varphi \varphi$ is thus a generalization of the tree-level result of Eq. (10) and takes automatically into account that the state $S$ has a finite width parametrized by the mass distribution $d_{S}(x)$, which naturally arises by considering the self-energy of the scalar propagator. Notice that the real part of the propagator is necessary in order Eq. (17) to hold: its neglection would spoil the correct normalization.

Evaluating the real and imaginary part at $x=M$ and neglecting their $x$-dependence, the distribution (21) is approximated by

$$
d_{S}^{\mathrm{bw}}(x) \simeq \frac{2 M}{\pi} \frac{I(M)}{\left(x^{2}-M^{2}\right)^{2}+(I(M))^{2}}
$$

which is the relativistic Breit-Wigner distribution for the resonance $S$, usually employed in theoretical and experimental studies. However, the distribution $d_{S}^{\mathrm{bw}}(x)$ neglects the real part of the loop diagram and consequently the normalization of Eq. (17) does not hold, implying that $d_{S}^{\text {bw }}(x)$ has to be normalized by hand. At the same time the mass $M$ does not coincide with the maximum of $d_{S}(x)$. Thus, we insist on that the usage of automatically normalized distribution emerging from propagators fulfilling Källen-Lehmann should be preferable.

\section{B. Application to the light scalar mesons $\sigma$ and $k$}

An interesting example for the one-channel case is the decay of the scalar meson $\sigma \equiv f_{0}(600)$. As reported by the PDG [5], experimental data are affected by large uncertainties both for the value of the mass, $M_{\sigma}=0.4-1.2 \mathrm{GeV}$, and the value of the Breit-Wigner width, $\Gamma_{\sigma}=0.6-1 \mathrm{GeV}$. The dominant channel, which we will consider here, is the decay into two pions, for which $M>2 m$.

By applying the formulas of Section II.A we show in the left panel of Fig. 目 the spectral functions $d_{\sigma}(x)$ of the $\sigma$-resonance for the two boundary cases of PDG, namely $M_{\sigma}=0.4 \mathrm{GeV}$ and $M_{\sigma}=1.2 \mathrm{GeV}$, respectively, for the coupling constant $g_{\sigma \pi \pi}=3 \mathrm{GeV}$.

The spectral function assumes different shapes for different values of the mass. While for $M_{\sigma}=1.2$, far from the threshold, the spectral function has a regular 'Breit-Wigner-like' form, in the case $M_{\sigma}=0.4 \mathrm{GeV}$ a distorted shape, with a narrow peak just above threshold, is visible [28]. The employed value of the coupling constant, $g_{\sigma \pi \pi}=3 \mathrm{GeV}$, serves as illustration and actually corresponds to a somewhat too narrow width. The increase of $g_{\sigma \pi \pi}$ leads, however, outside the range of validity of the normalization of Eq. (17) at one-loop level, see below.

The description of the scalar kaonic resonance $k$ follows the same line [26]. As shown in the right panel of Fig. [4 a strong deviation from the Breit-Wigner form is obtained when $g_{k \pi K}=6 \mathrm{GeV}$, corresponding to $\Gamma_{k}=0.38 \mathrm{GeV}$, while a less distorted shape is found for $g_{k \pi K}=3 \mathrm{GeV}$, for which $\Gamma_{k}=0.11 \mathrm{GeV}$.

At this point a short discussion on the definition of the mass of an unstable particle is needed: in the Breit-Wigner scheme, the mass of the particle is the value corresponding to the maximum of the spectral function and it is one of the parameter of the distribution (the second one is of course the width). In our scheme, using the spectral functions coming from the loop evaluation this is no more the case: the mass $M$ defined in Eq. (15) is again a parameter of the distribution (together with the coupling constant $g$ ) but it does not coincide with the maximum of the distribution. While for $M_{\sigma}=1.2 \mathrm{GeV}$ (far from threshold) the maximum of the spectral function occurs at $1.202 \mathrm{GeV}$, thus only slightly shifted from the mass, when $M_{\sigma}=0.4 \mathrm{GeV}$ the maximum of the spectral function (apart form the threshold enhancement peak) occurs at sizeable larger values respect to the mass, here $0.454 \mathrm{GeV}$ [29]. (Notice also that in the latter case the bare mass $M_{0}$ is $0.614 \mathrm{GeV}$, thus implying a strong influence of the pion loop to the sigma mass, see also Appendix A for a comparison of different 'masses'). Indeed, although the mass $M$ being the zero of the real part of the inverse propagator, see Eq. (15), is referred to as Breit-Wigner mass [25], the best fit to the full spectral function $d_{\sigma}(x)$ by using a Breit-Wigner form is obtained for a Breit-Wigner mass $M_{B W}$ coinciding with the maximum of the distribution. It is also remarkable that in some cases the spectral function has not a maximum, a part from

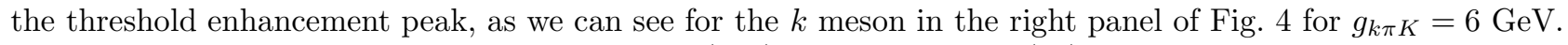

We now study closer the decay process $\sigma \equiv f_{0}(600) \rightarrow \pi \pi$ using Eq. (22) which implements the spectral function $d_{\sigma}(x)$. In Fig. [5 we compare the full and the tree-level decay rates for different values of the cut-off and of the coupling constants (a mass $M_{\sigma}=600 \mathrm{MeV}$ is used). 

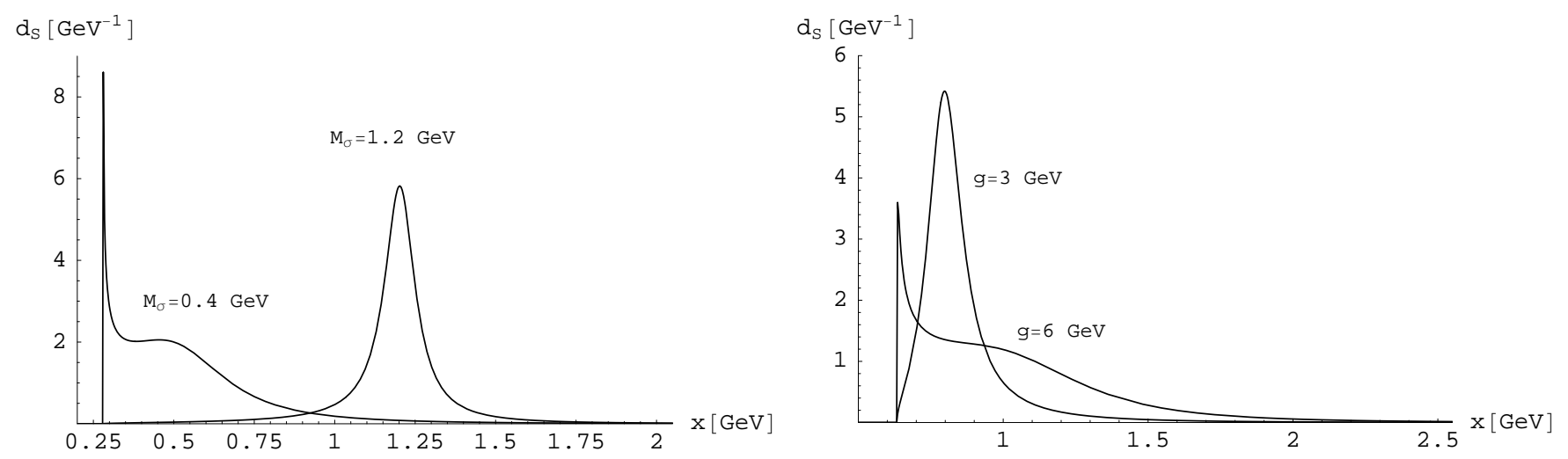

FIG. 4: Spectral functions of $\sigma$ and $k$.

Left panel: $\Lambda=1.5 \mathrm{GeV}, g=3 \mathrm{GeV}, M_{\sigma}=0.4 \mathrm{GeV}$ with a correspondent $\Gamma=0.242 \mathrm{GeV}$ and $M_{\sigma}=1.2 \mathrm{GeV}$ with a correspondent $\Gamma=0.113 \mathrm{GeV}$.

Right panel: $M_{k}=0.8 \mathrm{GeV}, \Lambda=1.5 \mathrm{GeV}, g=3 \mathrm{GeV}$ with a correspondent $\Gamma=0.112 \mathrm{GeV}$ and $g=6 \mathrm{GeV}$ with a correspondent $\Gamma=0.382 \mathrm{GeV}$

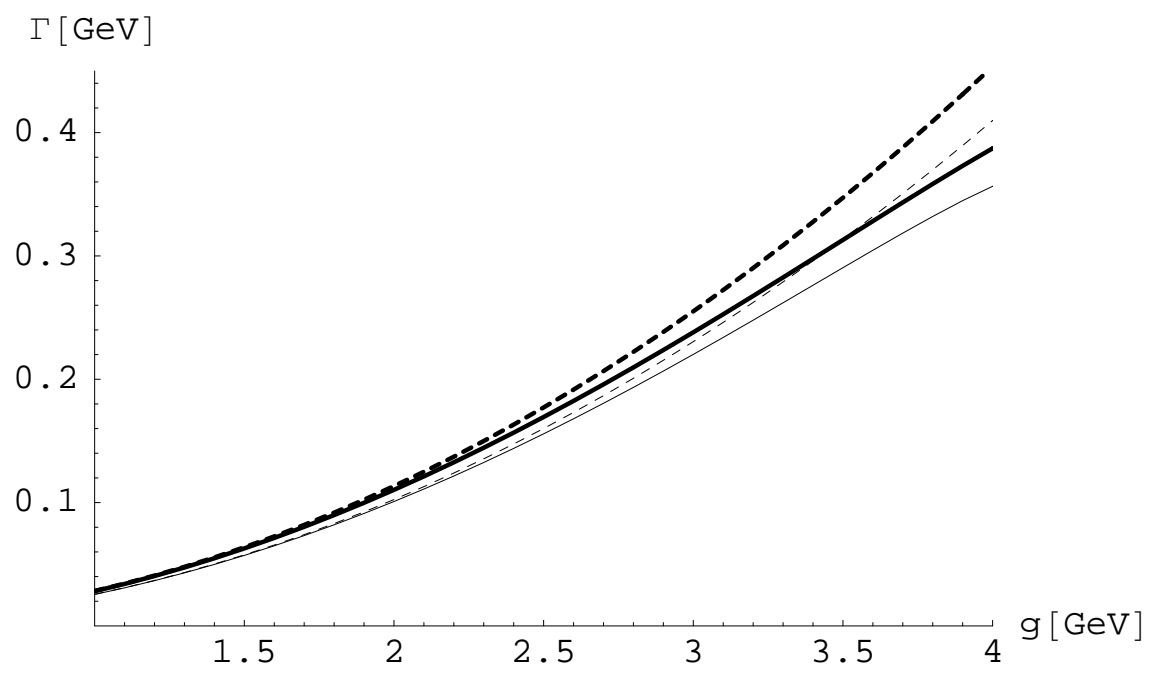

FIG. 5: The full (solid) and the tree-level (dashed) decay rates $\sigma \rightarrow \pi \pi$ are shown as function of the coupling constant. The cases $\Lambda=1$ and $\Lambda=2 \mathrm{GeV}$ correspond to thin and thick lines respectively.

As expected, there is not a strong dependence on the choice of the cut-off. Moreover the results obtained with our formulae are well in agreement with the tree-level results for small values of $g$ (since the spectral function tends to a delta function). Non-negligible differences instead occur for the larger values of $g$. This is due to a different analytic dependence of the two corresponding formulae from the coupling constant: while the tree-level expression depends quadratically on $g$, in the loop formula $g$ appears also in the distribution $d_{S}(x)$.

The limit of the validity of the employed one-loop level analysis is an important aspect which deserves further discussion. Namely, when the coupling constant is too large and the mass is not far from threshold the normalization condition of Eq. (17) is lost, see also the corresponding discussion in [22]. This fact means that higher orders must be taken into account to satisfy the Källen-Lehmann representation and thus to recover the correct normalization of Eq. (17). At the same time the violation of the normalization is a valid criterion to establish the limit of our study: for this reason in Fig. 5 we stop the plot at $g_{\sigma \pi \pi}=4 \mathrm{GeV}$ (corresponding to $\Gamma_{\sigma} \sim 400 \mathrm{MeV}$ ), in fact larger values imply $\int_{0}^{\infty} d_{S}(x) d x<1$. At this point the full decay width (using Eq. (22)) is already $\sim 60 \mathrm{MeV}$ smaller than the tree-level counterpart. A decay width of about $400 \mathrm{MeV}$ is on the low side for the $\sigma$ (see [21]). In Ref. [18] a width $150 \mathrm{MeV}$ larger is obtained. A study beyond one-loop level would then be necessary to evaluate the spectral function for larger coupling (i.e. larger width) and represents a possible outlook of the present work. Surely the overestimation of the tree-level formula keeps growing for increasing interaction strengths. Similar considerations hold for the $k$ meson. 


\section{SCALAR SPECTRAL FUNCTION: TWO-CHANNEL CASE}

\section{A. Definitions and properties}

We now consider two channels for the scalar resonance $S$ described by the Lagrangian density $\left(m_{2}>m_{1}\right)$ :

$$
\begin{aligned}
\mathcal{L}_{S}^{2}= & \frac{1}{2}\left(\partial_{\mu} S\right)^{2}-\frac{1}{2} M_{0}^{2} S^{2}+\frac{1}{2}\left(\partial_{\mu} \varphi_{1}\right)^{2}-\frac{1}{2} m_{1}^{2} \varphi_{1}^{2}+ \\
& \frac{1}{2}\left(\partial_{\mu} \varphi_{2}\right)^{2}-\frac{1}{2} m_{2}^{2} \varphi_{2}^{2}+g_{1} S \varphi_{1}^{2}+g_{2} S \varphi_{2}^{2} .
\end{aligned}
$$

The processes $S \rightarrow \varphi_{1} \varphi_{1}$ and $S \rightarrow \varphi_{2} \varphi_{2}$ correspond to the tree-level decay rates

$$
\begin{aligned}
& \Gamma_{S \varphi_{1} \varphi_{1}}^{\mathrm{t}-\mathrm{l}}\left(M_{0}\right)=\frac{p_{S \varphi_{1} \varphi_{1}}}{8 \pi M_{0}^{2}}\left[g_{S \varphi_{1} \varphi_{1}}\right]^{2} \theta\left(M_{0}-2 m_{1}\right), g_{S \varphi_{1} \varphi_{1}}=\sqrt{2} g_{1}, \\
& \Gamma_{S \varphi_{2} \varphi_{2}}^{\mathrm{t}-\mathrm{l}}\left(M_{0}\right)=\frac{p_{S \varphi_{2} \varphi_{2}}}{8 \pi M_{0}^{2}}\left[g_{S \varphi_{2} \varphi_{2}}\right]^{2} \theta\left(M_{0}-2 m_{2}\right), g_{S \varphi_{2} \varphi_{2}}=\sqrt{2} g_{2} .
\end{aligned}
$$

The propagator is modified by loops of $\varphi_{1}$ and $\varphi_{2}$, denoted as $\Sigma_{1}\left(p^{2}\right)$ and $\Sigma_{2}\left(p^{2}\right)$ and given by Eq. (7) for $m=m_{1}$ and $m=m_{2}$ respectively. A delocalization of the interaction, via a vertex-function $\Phi(y)$ and the corresponding Fourier-transform $f_{\Lambda}(q)=\int d^{4} y \Phi(y) e^{-i y q}$, is then introduced as in Eq. (8) for both channels in order to regularize the self-energy contributions. As a consequence, the tree-level results are modified as

$$
\begin{aligned}
\Gamma_{S \varphi_{1} \varphi_{1}}^{\mathrm{t}-1}\left(M_{0}\right) & =\frac{p_{S \varphi_{1} \varphi_{1}}}{8 \pi M_{0}^{2}}\left[g_{S \varphi_{1} \varphi_{1}} f_{\Lambda}\left(\vec{q}^{2}=p_{S \varphi_{1} \varphi_{1}}^{2}\right)\right]^{2} \theta\left(M_{0}-2 m_{1}\right) \\
\Gamma_{S \varphi_{2} \varphi_{2}}^{\mathrm{t}-1}\left(M_{0}\right) & =\frac{p_{S \varphi_{2} \varphi_{2}}}{8 \pi M_{0}^{2}}\left[g_{S \varphi_{2} \varphi_{2}} f_{\Lambda}\left(\vec{q}^{2}=p_{S \varphi_{2} \varphi_{2}}^{2}\right)\right]^{2} \theta\left(M_{0}-2 m_{2}\right),
\end{aligned}
$$

and the propagator as

$$
\Delta_{S}(x)=\frac{1}{x^{2}-M_{0}^{2}+R(x)+i I(x)+i \varepsilon} .
$$

where

$$
R(x)=g_{S \varphi_{1} \varphi_{1}}^{2} \operatorname{Re}\left[\Sigma_{1}\left(x=\sqrt{p^{2}}\right)\right]+g_{S \varphi_{2} \varphi_{2}}^{2} \operatorname{Re}\left[\Sigma_{2}\left(x=\sqrt{p^{2}}\right)\right]
$$

and

$$
\begin{aligned}
I(x) & =g_{S \varphi_{1} \varphi_{1}}^{2} \operatorname{Im}\left[\Sigma_{1}\left(x=\sqrt{p^{2}}\right)\right]+g_{S \varphi_{2} \varphi_{2}}^{2} \operatorname{Im}\left[\Sigma_{2}\left(x=\sqrt{p^{2}}\right)\right] \\
& =x \Gamma_{S \varphi_{1} \varphi_{1}}^{\mathrm{t}-1}(x)+x \Gamma_{S \varphi_{2} \varphi_{2}}^{\mathrm{t}-1}(x) .
\end{aligned}
$$

In the last equation the optical theorem has been used. The mass $M$ of the state $S$ is given by $M^{2}-M_{0}^{2}+R(M)=0$. Again, we have two cases:

(i) $M<2 m_{1}$ : the distribution $d_{S}(x)$ takes the form $Z \delta(x-M)$ for $x<2 m_{1}$. The discussion is similar to the one-channel case. Eq. (19) is still valid. At threshold $2 m_{1}$ the continuum starts.

(ii) $M>2 m_{1}$ : as in Eq. (21) the distribution is

$$
d_{S}(x)=\frac{2 x}{\pi} \frac{I(x)}{\left(x^{2}-M_{0}^{2}+R(x)\right)^{2}+I(x)^{2}} .
$$

It vanishes for $M<2 m_{1}$. At $x=2 m_{2}$ the second channel opens.

In the case (ii) we have a resonant state. The decay rates into the two channels $S \rightarrow \varphi_{1} \varphi_{1}$ and $S \rightarrow \varphi_{2} \varphi_{2}$ are given by the integrals:

$$
\Gamma_{S \varphi_{1} \varphi_{1}}=\int_{0}^{\infty} d x d_{S}(x) \Gamma_{S \varphi_{1} \varphi_{1}}^{\mathrm{t}-1}(x), \Gamma_{S \varphi_{2} \varphi_{2}}=\int_{0}^{\infty} d x d_{S}(x) \Gamma_{S \varphi_{2} \varphi_{2}}^{\mathrm{t}-1}(x) .
$$

A particularly interesting case takes place when $2 m_{1}<M<2 m_{2}$ : while the tree-level result for $S \rightarrow \varphi_{2} \varphi_{2}$ vanishes, we find that $\Gamma_{S \varphi_{2} \varphi_{2}}$ is not zero. In this case the tree-level approximation is absolutely not applicable: the particle 

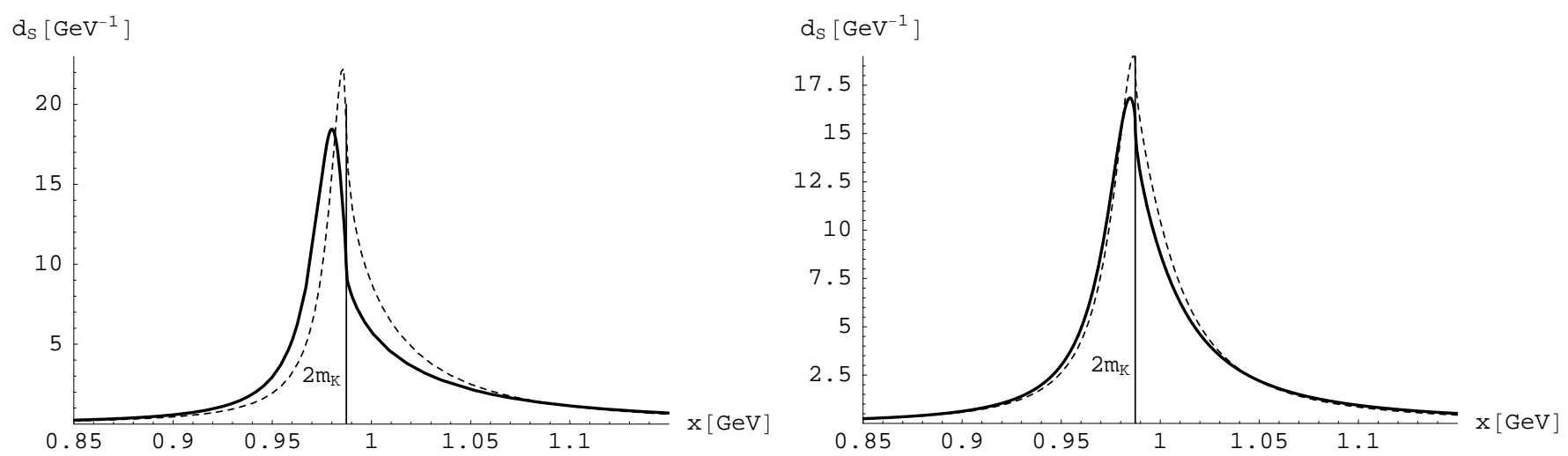

FIG. 6: Left panel: spectral function of $f_{0}$ within our formalism (solid line) and using the Flatte' distribution (dashed line). Parameters are: $\Lambda=1.5 \mathrm{GeV}, g_{f_{0} K \bar{K}}=3 \mathrm{GeV}$ with a correspondent total decay rate $\Gamma=0.058 \mathrm{GeV}$.

Right panel: spectral function of $a_{0}$ within our formalism (solid line) and using the Flatte' distribution (dashed line). Parameters are: $\Lambda=1.5 \mathrm{GeV}, g_{f_{0} K \bar{K}}=3 \mathrm{GeV}$, with a correspondent total decay rate $\Gamma=0.048 \mathrm{GeV}$.

$S$ does decay in virtue of the high-mass tail of its distribution. A physical example is well-known: the resonances $f_{0}(980)$ and $a_{0}(980)$ have a non-zero decay rate into $\bar{K} K$, although their masses are below the threshold $2 m_{K}$. Clearly, a sizable decay rate $\Gamma_{S \varphi_{2} \varphi_{2}}$ is obtained only when $M$ is close to threshold. A generalization to the present definitions to $N$ channels is straightforward [22].

When applying the decay formulas (35) it is however important to verify numerically that the normalization of the distribution $d_{S}(x)$ holds: in fact, as discussed in Section II.B, only in this case the formalism is self-consistent.

\section{B. Application to $a_{0}(980)$ and $f_{0}(980)$}

In this subsection we study the spectral functions of the scalar mesons $a_{0} \equiv a_{0}(980)$ and $f_{0} \equiv f_{0}(980)$, whose masses are $M_{a_{0}}=984.7 \pm 1.2 \mathrm{MeV}$ and $M_{f_{0}}=980 \pm 10 \mathrm{MeV}$ [ 5$]$. For both resonances two decays have been observed: $a_{0} \rightarrow \pi \eta, a_{0} \rightarrow K \bar{K}$ and $f_{0} \rightarrow \pi \pi, f_{0} \rightarrow K \bar{K}$. Notice that both masses are below threshold of kaon-antikaon production, $M_{a_{0}, f_{0}}<2 M_{K}=987.3 \mathrm{MeV}$, thus the decay of both resonances in $K \bar{K}$ vanishes at tree-level, while experimentally it was seen for both $a_{0}$ and $f_{0}$ states.

For definiteness we use the following ratios obtained in the experimental analysis of [4]

$$
\frac{g_{f_{0} K \bar{K}}^{2}}{g_{f_{0} \pi \pi}^{2}}=4.21 \pm 0.46, \frac{g_{f_{0} K \bar{K}}^{2}}{g_{a_{0} K \bar{K}}^{2}}=2.15 \pm 0.40, \frac{g_{a_{0} \pi \eta}^{2}}{g_{a_{0} K \bar{K}}^{2}}=0.75 \pm 0.11
$$

therefore leaving us with only one free parameter, chosen to be $g_{f_{0} K \bar{K}}$. Although experimental uncertainties are still large, the results of Eq. (36) are qualitative similar to various studies, see [24] and Refs. therein, pointing to a large $K \bar{K}$ coupling for both resonances with a particular enhancement for $f_{0}$ (see [4, 10, 11, 15] and Refs. therein for spectroscopic interpretations).

For the typical value $g_{f_{0} K \bar{K}}=3 \mathrm{GeV}$ we report in Fig. 6 the spectral functions of $a_{0}$ and $f_{0}$. There is a large probability, $\sim 50 \%$, in both cases, that these two mesons have a mass larger than the threshold of production $2 m_{K}$ and therefore the tree-level forbidden decay occurs. In the same figure we compare our distribution with the Flattè one [23, 24], which is usually employed for the $a_{0}$ and $f_{0}$ mesons. At variance from our distribution, Flattè distribution must be normalized by hand. The two distributions are quite similar, only for the $f_{0}$ the values of the mass corresponding to the maximum of the distributions are slightly different. This is due to the strong coupling of $f_{0}$ to kaons and, as already argued by Achasov [22], the meson loop distributions coincide with the Flattè ones only in the limit of weak coupling.

In Fig. 7 we show the decay rates $f_{0} \rightarrow \pi \pi, f_{0} \rightarrow K \bar{K}$ and $a_{0} \rightarrow \pi \eta, a_{0} \rightarrow K \bar{K}$ as function of $g_{f_{0} K \bar{K}}$. The dashed areas in both plots correspond to the total decay rate of $f_{0}$ and $a_{0}$ as indicated by the PDG (notice, however, that in a note in PDG it is specified that the real width could be larger). To get agreement between our theoretical total decay rates and the measured ones, $g_{f_{0} K \bar{K}}$ has to lye between 3-4 GeV. The outcoming branching ratio $\Gamma_{a_{0} K \bar{K}} / \Gamma_{a_{0} \pi \eta}$ is $\sim 0.3$-0.4, larger than the PDG average 0.183 \pm 0.024 , while the obtained ratio $\Gamma_{f_{0} \pi \pi} /\left(\Gamma_{f_{0} \pi \pi}+\Gamma_{f_{0} K \bar{K}}\right)$ is $\sim 0.48-0.56$ 

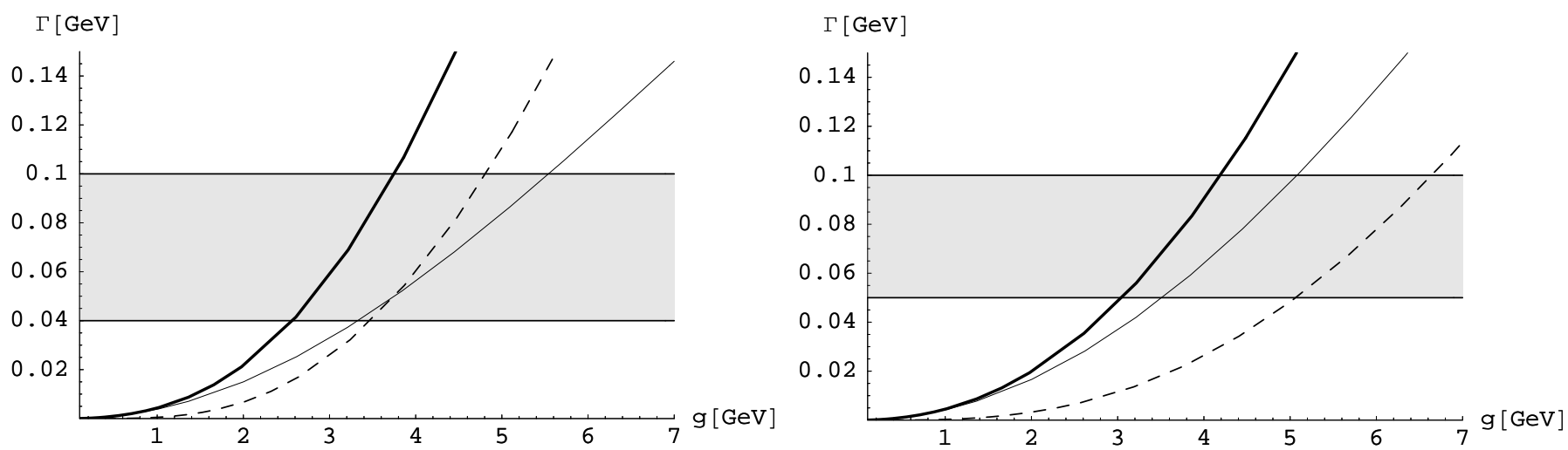

FIG. 7: Left panel: decay rates of $f_{0}$ as functions of the coupling constant $g$. The thin solid line corresponds to the decay into two pions, the dashed line to the decay into two kaons and the thick solid line is the sum of the two decay rates.

Right panel: decay rates of $a_{0}$ as functions of the coupling constant $g$. The thin solid line corresponds to the decay into pion and eta, the dashed line to the decay into two kaons and the thick solid line is the sum of the two decay rates.

in qualitative agreement with the (not bold) results listed in PDG. Notice furthermore that the $f_{0}$ mesons turns out to have typically a larger width than $a_{0}$ meson in agreement with Ref. 24].

We finally comment on a possible tetraquark unified interpretation of the light scalar mesons as presented in Ref. [11]. A too small decay constant $g_{f_{0} K \bar{K}}$ would also imply a by far too narrow $\sigma$ and $k$ mesons (related by ClebshGordon coefficients [11]), thus against a tetraquark nonet. On the contrary, $g_{f_{0} K \bar{K}}$ between $3-4 \mathrm{GeV}$ is in agreement with a tetraquark nonet below $1 \mathrm{GeV}$, although problems, such as a too narrow $k$, persist, see discussions in [11, 15]. Such a strong coupling in the $K \bar{K}$-channel implies that virtual cloud of kaon-antikaon pairs plays an important role, in particular for the $f_{0}$ resonance. A heuristic indicator of the mesonic cloud can be given by the quantity $Z_{\bar{K} K}=\left(1+\frac{1}{2 M_{S}}\left(\frac{d R_{\overline{S K} K}}{d x}\right)_{x=M_{S}}\right)^{-1}$ where $R_{S \bar{K} K}=g_{S \bar{K} K}^{2} R e\left[\Sigma_{K K}(x)\right]$ (with $\left.S=f_{0}, a_{0}\right)$ refers to the kaonic loop only. As discussed in Section II in the subthreshold case (which applies to the kaonic channel here) the quantity $(1-Z)$ varies between 0 and 1 and measures the mesonic cloud dressing of the original bare resonance $S$. In the $f_{0}$ case, by using Eq. (36) together with $g_{f_{0} K \bar{K}}=3 \mathrm{GeV}$, one finds $\left(1-Z_{\bar{K} K}\right)=0.38$, hence implying a $38 \%$ of kaonic cloud. This number increases for increasing coupling strength $g_{f_{0} K \bar{K}}$. This discussion confirms the interpretation put forward in Ref. [2], where the light scalar mesons posses a tetraquark core but are dressed by kaonic clouds.

\section{SUMMARY AND CONCLUSIONS}

In this work we studied the spectral functions of scalar mesons in one- and two-channel cases suitable for the description of light scalar mesons below $1 \mathrm{GeV}$. We have computed, by using Lagrangians with non-derivative couplings, the propagators of scalar mesons at one-loop level. They satisfy for large ranges of parameters the Källen-Lehmann representation, therefore implying normalized spectral functions. In this way a correct definition of decay amplitudes, weighted over the spectral function, is formulated: the finite-width effects are automatically taken into account. The resulting decay rates are smaller than the tree-level ones with increasing mismatch for increasing interaction strength. On the other hand, a sub-threshold tree-level forbidden decay, such as the $K \bar{K}$ mode for $a_{0}(980)$ and $f_{0}(980)$, becomes large.

The resulting spectral functions for the $\sigma$ and $k$ mesons may deviate consistently from the Breit-Wigner form. The Flattè distribution, although it approximates to a good level of accuracy the $a_{0}(980)$ and $f_{0}(980)$ spectral functions, emerges as a small-coupling limit of our more general spectral function.

As stressed by Achasov [22] it is important to use distributions satisfying Källen-Lehmann representations in experimental and theoretical studies. We thus believe that the use of distributions obtained from quantum field theoretical models fulfilling the correct normalization requirements can be helpful to correctly disentangle the nature of the scalar states. Future studies with derivative couplings, mixing effects and $\phi$-decays represent a possible interesting outlook.

Acknowledgements G.P. acknowledges financial support from INFN. 


\section{APPENDIX A: LOOP CONTRIBUTIONS}

Here we report basic formulas for the loop diagram of Eq. (7) drawn in Fig.1 for the vertex function $f_{\Lambda}(q)=f_{\Lambda}\left(\vec{q}^{2}\right)$. By evaluating the residua one obtains the one-dimensional integral

$$
\Sigma\left(x=p^{2}\right)=\frac{1}{2 \pi^{2}} \int_{0}^{\infty} \mathrm{dw} \frac{w^{2} f_{\Lambda}^{2}(w)}{\sqrt{w^{2}+m^{2}}\left(4\left(w^{2}+m^{2}\right)-x^{2}\right)}
$$

which can be easily evaluated numerically for each well-behaved $f_{\Lambda}(w)$. We remind that within our conventions $f_{\Lambda}(0)=1$ and that $w=|\vec{k}|$; Eq. (A1) refers to a 3d-vertex function. In [22] the form $f_{\Lambda}(w)=\theta(\Lambda-w)$ is used and the limit $\Lambda \rightarrow \infty$ is taken. As described in the text, we did not follow this procedure but we used definite form(s) for the vertex function $f_{\Lambda}(w)$. As remarked in the text, we performed the calculations also with different forms for $f_{\Lambda}(w)$ (different power form and exponential functions): the precise form of the cutoff function does not affect the physical picture.

When the scalar state $S$ couples to two particles of masses $m_{1}$ and $m_{2}$ the loop contribution is modified as following:

$$
\Sigma(x)=\frac{1}{4 \pi^{2}} \int_{0}^{\infty} \mathrm{dw} \frac{w^{2}\left(\sqrt{w^{2}+m_{1}^{2}}+\sqrt{w^{2}+m_{2}^{2}}\right) f_{\Lambda}^{2}(w)}{\sqrt{w^{2}+m_{1}^{2}} \sqrt{w^{2}+m_{2}^{2}}\left(\left(\sqrt{w^{2}+m_{1}^{2}}+\sqrt{w^{2}+m_{2}^{2}}\right)^{2}-x^{2}\right)} .
$$

The choice $f_{\Lambda}(w)=\left(1+w^{2} / \Lambda^{2}\right)^{-1}$ with $\Lambda=1-2 \mathrm{GeV}$ has been used in this work.

In relation to the mass definition of Section II.B we report and compare in Table 1 the mass $M$ defined in Eq. (15), the bare mass $M_{0}$, the maximum $M_{\max }$ of the distribution $d_{S \equiv \sigma}(x)$ and the average mass $\langle M\rangle=\int_{0}^{\infty} \mathrm{dx} x d_{S}(x)$. We use $m=m_{\pi}, g_{\sigma \pi \pi}=3 \mathrm{GeV}, \Lambda=1.5 \mathrm{GeV}$.

Tab.1: Comparison of 'masses'

\begin{tabular}{|l|l|l|l|}
\hline$M$ & $M_{0}$ & $M_{\max }$ & $\langle M\rangle$ \\
\hline 0.4 & 0.61 & 0.45 & 0.54 \\
\hline 0.6 & 0.71 & 0.62 & 0.65 \\
\hline 0.8 & 0.86 & 0.81 & 0.82 \\
\hline 1 & 1.03 & 1.00 & 1.01 \\
\hline
\end{tabular}

As expected, the larger the mass, the smaller the differences among the various mass-like quantities.

\section{APPENDIX B: SPECTRAL FUNCTION AS 'MASS DISTRIBUTION': AN INTUITIVE DISCUSSION}

We present an intuitive argument for the correctness of interpretation of the spectral function $d_{S}(x)$ as the "mass distribution' of the state $S$. To this end we introduce two scalar fields $A$ and $B$, the first massless and the second with $M_{B}>M_{S}$ and write down the interaction Lagrangian

$$
L=c B A S+g S \varphi^{2} .
$$

(for the following discussion the 'delocalization' of Eq. (8) is not important). We suppose that the interaction strength $c$ is small enough to allow a tree-level analysis for the decay of the state $B$. The term $c B A S$ generates the decay process $B \rightarrow A S$, which reads (at tree-level)

$$
\Gamma_{B A S}^{\mathrm{t}-\mathrm{l}}\left(M_{B}\right)=\frac{p_{B A S}}{8 \pi M_{B}^{2}}[c]^{2} .
$$

However, when $g \neq 0$ the state $S$ decays into $\varphi \varphi$, that is the state $S$ is not an asymptotic state. Physically, we observe a tree-body decay $B \rightarrow A \varphi \varphi$, whose decay-rate reads:

$$
\Gamma_{B A \varphi \varphi}^{\mathrm{t}-1}\left(M_{B}\right)=\int_{0}^{M_{B}} \Gamma_{B A S}^{\mathrm{t}-1}\left(M_{B}\right) d_{S}(x) d x
$$

The tree-body decay is decomposed into two steps: $B \rightarrow A S$ and $S \rightarrow \varphi \varphi$. The quantity $\Gamma_{B A S}^{\mathrm{t}-\mathrm{l}}\left(M_{B}\right)$ represents the probability for $B \rightarrow A S$ (at a given mass $x$ for the state $S$ ) and $d_{S}(x) d x$ is the corresponding weight, i.e. the probability 
that the resonance $S$ has a mass between $x$ and $x+d x$. In this example $d_{S}(x)$ emerges naturally as a mass distribution, correctly normalized, for the scalar state $S$. Furthermore, notice that in virtue of the limit $d_{S}(x)=\delta\left(M_{-}-M_{S}\right)$ for $g \rightarrow 0$ one has

$$
\Gamma_{B A \varphi \varphi}^{\mathrm{t}-\mathrm{l}}\left(M_{B}\right)=\Gamma_{B A S}^{\mathrm{t}-\mathrm{l}}\left(M_{B}\right) \text { for } g \rightarrow 0 .
$$

In fact, if $g$ is very small the state $S$ is long-living and the equation (B2) is recovered. The present analysis also shows that studies on the tree-body decay of the $\phi$ meson can be consistent only if propagators satisfying the Källen-Lehmann representation are used.

[1] C. Amsler and N. A. Tornqvist, Phys. Rept. 389, 61 (2004).

[2] F. E. Close and N. A. Tornqvist, J. Phys. G 28, R249 (2002) arXiv:hep-ph/0204205;

[3] R. L. Jaffe, Phys. Rept. 409 (2005) 1 [Nucl. Phys. Proc. Suppl. 142 (2005) 343] arXiv:hep-ph/0409065. R. L. Jaffe, arXiv:hep-ph/0701038. M. R. Pennington, arXiv:hep-ph/0703256. N. N. Achasov, arXiv:hep-ph/0609261.

[4] D. V. Bugg, Eur. Phys. J. C 47 (2006) 57 arXiv:hep-ph/0603089. D. V. Bugg, Eur. Phys. J. C 47 (2006) 45 arXiv:hep-ex/0603023.

[5] W. M. Yao et al. [Particle Data Group], J. Phys. G 33 (2006) 1.

[6] C. Amsler and F. E. Close, Phys. Lett. B 353, 385 (1995) arXiv:hep-ph/9505219; C. Amsler and F. E. Close, Phys. Rev. D 53, 295 (1996) arXiv:hep-ph/9507326.

[7] R. L. Jaffe, Phys. Rev. D 15 (1977) 267. R. L. Jaffe, Phys. Rev. D 15 (1977) 281. R. L. Jaffe and F. E. Low, Phys. Rev. D 19, 2105 (1979).

[8] M. B. Voloshin and L. B. Okun, JETP Lett. 23, 333 (1976) [Pisma Zh. Eksp. Teor. Fiz. 23, 369 (1976)]. K. Maltman and N. Isgur, Phys. Rev. Lett. 50, 1827 (1983). K. Maltman and N. Isgur, Phys. Rev. D 29, 952 (1984).

[9] L. Maiani, F. Piccinini, A. D. Polosa and V. Riquer, Phys. Rev. Lett. 93 (2004) 212002 arXiv:hep-ph/0407017.

[10] N. N. Achasov and V. N. Ivanchenko, Nucl. Phys. B 315 (1989) 465.

[11] F. Giacosa, Phys. Rev. D 74 (2006) 014028 arXiv:hep-ph/0605191.

[12] F. E. Close and A. Kirk, Eur. Phys. J. C 21, 531 (2001) arXiv:hep-ph/0103173]. W. J. Lee and D. Weingarten, Phys. Rev. D 61, 014015 (2000) arXiv:hep-lat/9910008; M. Strohmeier-Presicek, T. Gutsche, R. Vinh Mau and A. Faessler, Phys. Rev. D 60, 054010 (1999) arXiv:hep-ph/9904461]. F. Giacosa, T. Gutsche and A. Faessler, Phys. Rev. C 71, 025202 (2005) arXiv:hep-ph/0408085. F. Giacosa, T. Gutsche, V. E. Lyubovitskij and A. Faessler, Phys. Rev. D 72, 094006 (2005) arXiv:hep-ph/0509247. F. Giacosa, T. Gutsche, V. E. Lyubovitskij and A. Faessler, Phys. Lett. B 622, 277 (2005) arXiv:hep-ph/0504033. G. Mosconi, private communications.

[13] D. Black, A. H. Fariborz, S. Moussa, S. Nasri and J. Schechter, Phys. Rev. D 64 (2001) 014031 arXiv:hep-ph/0012278.

[14] A. H. Fariborz, R. Jora and J. Schechter, Phys. Rev. D 72 (2005) 034001 arXiv:hep-ph/0506170. A. H. Fariborz, Int. J. Mod. Phys. A 19 (2004) 2095 arXiv:hep-ph/0302133. A. H. Fariborz, Phys. Rev. D 74 (2006) 054030 arXiv:hep-ph/0607105. M. Napsuciale and S. Rodriguez, Phys. Rev. D 70 (2004) 094043 arXiv:hep-ph/0407037.

[15] F. Giacosa, Phys. Rev. D 75 (2007) 054007 arXiv:hep-ph/0611388.

[16] E. van Beveren, F. Kleefeld, G. Rupp and M. D. Scadron, Mod. Phys. Lett. A 17 (2002) 1673 arXiv:hep-ph/0204139. M. D. Scadron, G. Rupp, F. Kleefeld and E. van Beveren, Phys. Rev. D 69 (2004) 014010 [Erratum-ibid. D 69 (2004) 059901] arXiv:hep-ph/0309109.

[17] P. Minkowski and W. Ochs, Nucl. Phys. Proc. Suppl. 121, 123 (2003) arXiv:hep-ph/0209225;

[18] I. Caprini, G. Colangelo and H. Leutwyler, Phys. Rev. Lett. 96 (2006) 132001 arXiv:hep-ph/0512364. G. Colangelo, J. Gasser and H. Leutwyler, Nucl. Phys. B 603 (2001) 125 arXiv:hep-ph/0103088.

[19] J. A. Oller, E. Oset and J. R. Pelaez, Phys. Rev. D 59, 074001 (1999) [Erratum-ibid. D 60, 099906 (1999)] arXiv:hep-ph/9804209.

[20] J. R. Pelaez, Phys. Rev. Lett. 92 (2004) 102001 arXiv:hep-ph/0309292. Mod. Phys. Lett. A 19 (2004) 2879 arXiv:hep-ph/0411107. J. R. Pelaez and G. Rios, Phys. Rev. Lett. 97 (2006) 242002 arXiv:hep-ph/0610397.

[21] M. Ishida, Prog. Theor. Phys. 101 (1999) 661 arXiv:hep-ph/9902260. Z. H. Guo, L. Y. Xiao and H. Q. Zheng, arXiv:hep-ph/0610434.

[22] N. N. Achasov and A. V. Kiselev, Phys. Rev. D 70 (2004) 111901 arXiv:hep-ph/0405128.

[23] S. M. Flatte, Phys. Lett. B 63 (1976) 228.

[24] V. Baru, J. Haidenbauer, C. Hanhart, A. Kudryavtsev and U. G. Meissner, Eur. Phys. J. A 23 (2005) 523 arXiv:nucl-th/0410099.

[25] N. A. Tornqvist, Z. Phys. C 68 (1995) 647 arXiv:hep-ph/9504372. M. Boglione and M. R. Pennington, Phys. Rev. D 65 (2002) 114010 arXiv:hep-ph/0203149.

[26] The resonance $k(800)$ is now listed in the compilation of the Particle Data Group [5] but it still needs confirmation and is omitted from the summary table. The resonance is also found in many recent theoretical and experimental works ([4, 19], E. Van Beveren, T. A. Rijken, K. Metzger, C. Dullemond, G. Rupp and J. E. Ribeiro, Z. Phys. C 30 (1986) 615. S. Ishida, M. Ishida, T. Ishida, K. Takamatsu and T. Tsuru, Prog. Theor. Phys. 98 (1997) 621 arXiv:hep-ph/9705437. D. Black, A. H. Fariborz, F. Sannino and J. Schechter, Phys. Rev. D 58 (1998) 054012 arXiv:hep-ph/9804273 and Refs. therein.) 
[27] For a covariant vertex function $f_{\Lambda}(q)$ the decay-amplitude takes the form $\left[g_{S \phi \phi} f_{\Lambda}\left(q^{0}=0, \mathbf{q}^{2}=p_{S \varphi \varphi}^{2}\right)\right]$

[28] In the limit $M_{\sigma} \rightarrow 2 m_{\pi}$ the spectral function $d_{S}(x) \sim 1 / I(x) \rightarrow \infty$ for $x \rightarrow 2 m_{\pi}$ due to the threshold enhancement.

[29] Recent theoretical works [18] find a sigma mass at around $450 \mathrm{MeV}$, thus not far from threshold in the lower side of the PDG data: this is indeed the case of irregular form for the spectral function of this resonance, for which care is needed. 